\title{
Learning by Doing and Fragmentation
}

\author{
Eric Bond* and Yan $\mathrm{Ma}^{\dagger}$ \\ Vanderbilt University and Kobe University \\ First version: November
}

\begin{abstract}
*Department of Economics, Vanderbilt University,VU Station B\#351819, 2301Vanderbilt Place, Nashville, TN 27235, USA. Tel: (+1) 615-322-2388. E-mail: eric.bond@Vanderbilt.Edu;

${ }^{\dagger}$ Graduate School of Business Administration, Kobe University, Rokkodai-cho 2-1, Nada-ku, Kobe 657-8501, Japan. Tel: +81-78-803-6980; Fax: +81-78-803-6977; E-mail: mayan003@kobe-u.ac.jp.
\end{abstract}




\section{Introduction}

Jones and Kierzkowski (1991) were the first to focus on the "fragmentation" of production, where the reduction of communication and transportation costs has made it possible to break up the production of a good into different fragments that are produced in different locations around the world. This phenomenon, which also has been referred to as vertical specialization and international outsourcing, has been widely studied in the theoretical literature. ${ }^{1}$ The focus in the literature on fragmentation has been primarily static, in that it examines the factors that are responsible for the existence of fragmentation and its implications for the pattern of trade and income distribution.

Our focus in this paper is on the dynamics of the fragmentation process. A number of examples exist in which countries have gone from producing only a small part of the production chain to moving up along the production chain to produce more complex parts through learning by doing. Hobday (1995) described how Anam Industrial (South Korea) entered the chip packaging business by first learning the art of the assembly of simple transistors and discrete devices and then integrated circuits for export to the US. The growing packaging demand from the US in the 1980s pushed Anam into developing process-engineering skills for more complex products. Other examples are the success of Taiwanese machine tool firms (Amsden, 1977) and Bangladesh textile industry (Bond, Jnes and Wang (2005)).

To capture this dynamic fragmentation process, we develop a simple two sector Ricardian model of trade that highlights two relationships between knowledge accumulation and the production of "fragments". One is the effect of current output of a fragment on the future stock of knowledge, which is called "learning by doing" effect. The other is the benefit of accumulated knowledge to the productivity of the individual fragments, which is called "productivity improvement effect". Knowledge is treated as the public good in the sector, and hence forward and backward knowledge linkage emerges. Forward knowledge linkage occurs when output of a simple fragment contributes to knowledge that raises the productivity of a more complex fragment, while a backward knowledge linkage occurs when output of a complex fragment contributes to productivity of a simpler fragment.

Our paper is not the first study that emphasizes the role of externality from learning-bydoing. Young (1991) pointed out the role of learning-by-doing in a Ricardian model of trade, where more complicated goods have higher unit labor requirements but generate greater gains from learning-by-doing. In addition, free trade results in loss of learning intensive industries

\footnotetext{
${ }^{1}$ In the existing theoretical literature on fragmentation, there are many good studies. Some studies emphasize the role of service links in connecting fragmented production blocks and scale economies, for example, Jones and Kierzkowski $(1990,2001)$ and Golub, Jones, and Kierzkowski (2007). Some papers discuss the relationship between fragmentation and comparative advantage, for example, Dearoff (2001), Bond (2005), Grossman and Rossi-hansberg (2008). There are also some studies that highlight the contracting issues caused by fragmentation, for example, Grossmand and Helpman (2002), Antras and Helpman (2004), and Bond (2008). Some other studies focus on the tasks and matching process, for example, Grossman and Rossi-hansberg (2010), Dei (2010) and Nighenthi, Ma and Dei (2011).
} 
in his model. In contrast to his model, the closer the "fragment" is to the production frontier of developing countries, the greater gains it benefits from learning-by-doing in our model. Goh and Wan (2005) compare free trade with fragmentation to autarky with learning effects. Our study differs in that developing countries expand the production range of "fragments" and learning by performing the current range of "fragments" leads to having comparative advantage in the other range. Bond, Jones and Wang (2005) pointed out that trade liberalization plays a role in the growth of developing countries and comparative advantage in goods evolves due to the accumulation of knowledge. Their focus is trade liberalization and learning effects arise from exporting. Learning in our model arises from performing the production of the current range of fragments and leads to knowledge accumulation which shifts the comparative advantage to more complicated fragments.

The framework is developed in section 2. In section 3, we discuss the competitive equilibrium with trade. The social planner's problem is considered in section 4 . Section 5 analyzed dynamics of the socially optimal plan.

\section{$2 \quad$ Model}

We consider a two country, two good Ricardian model of international trade. We refer to the two countries as home and foreign, and denote foreign variables with a "*". The labor endowments of the home and foreign countries are denoted by $L$ and $L^{*}$, respectively. Preferences for the goods are assumed to take a quasi-linear form, $U=C_{1}+u\left(C_{2}\right)\left(U^{*}=C_{1}^{*}+u^{*}\left(C_{2}^{*}\right)\right)$, with $u\left(u^{*}\right)$ a strictly concave function. Good 1 is a standardized good that has only one stage, and whose unit labor requirements are given by $a\left(a^{*}\right)$ in the home (foreign) country. Sector 2 has a production technology that requires a continuum of "fragments" indexed by $s \in[0,1]$, with each unit of good 2 requiring input of 1 unit of each of the fragments. The foreign country is assumed to have exhausted all of its benefits from learning by doing in production of good 2 , so its unit labor requirements, $b^{*}(s)$ will be unchanging over time.

The home country unit labor requirements for the fragments of good 2 , in contrast, will be changing over time as it gains experience in the production of good 2. We assume that the current state of production experience can be summarized by $K$, which can be interpreted as stock of knowledge. In order to fully specify the dynamics of relationships between the stock of knowledge and the production fragments in sector 2, we need two relationships. One is the benefit of accumulated knowledge to the productivity of the individual fragments, and the other is the effect of current output of a fragment on the future stock of knowledge. We examine each of these relationships in turn.

The home unit labor requirements technology for segment $s$ will be expressed as $b(s, K)$. The indexing of fragments should be thought of as ordering fragments in increasing complexity, with more complex products requiring greater skill on the part of labor. For example, the lowest values of $s$ might correspond to simple assembly operations. Intermediate values could represent more sophisticated production activities, and the highest levels would involve engineering improvements and designs. The following assumptions impose restrictions on the home country chain of comparative advantage, which are intended to capture the notion that 
higher values of $s$ are associated with more complex fragments that require greater knowledge to produce more efficiently.

Assumption 1:

(a) $\frac{b(s, K)}{b^{*}(s)}$ is continuous and increasing in $s$ for all $K$

(b) for a given level of knowledge, there exists a critical level of complexity, $s^{b}(K)$, such that

$b_{K}(s, K)<0$ for $s>s^{b}(K)$ and $b_{K}(s, K)=0$ for $s \leq s^{b}(K)$, where $s^{b}(K)$ is increasing in $K$

(c) at the initial stock of knowledge $K_{0}, \frac{b\left(0, K_{0}\right)}{b^{*}(0)}<\frac{a}{a^{*}}<\frac{b\left(1, K_{0}\right)}{b^{*}(1)}$

Assumption (1a) associates the fragment index $s$ with the home country unit labor requirement relative to the foreign unit labor requirement. Given the Ricardian framework, this ratio can be interpreted as a chain of comparative advantage in the production fragments, with home country comparative advantage decreasing in $s$ for all levels of knowledge. Part (b) puts a bound on the degree of productivity improvement for each fragment by assuming that for a given stock of knoledge, only fragments exceeds $s^{b}(K)$ will benefit from learning. The threshold $s^{b}(K)$ is increasing in $K$, which means that for more complex fragments, productivity gains from learning will be exhausted at a higher value of $K$. Part (c) places the standardized good in the interior of the chain of comparative advantage: the home country will have lesser relative labor requirement (greater) in the simplest (most complex) fragments than it does in the standardized goods.

Figure 1 illustrates the implications of Assumption 1 for the relative unit labor requirements. At knowledge stock $K_{0}$ only the least complex fragment has exhausted the gains from learning, while at $K_{\max }$ the gains from knowledge have been exhausted for all fragments. At $K_{1}$ productivity gains are still possible for $s>s^{b}\left(K_{1}\right)$, reflecting Assumption 1b. Figure 1 illustrates a case in which $\frac{b\left(1, K_{\max }\right)}{b^{*}(1)}>\frac{a}{a^{*}}$, although our assumptions are also consistent with a case in which the home country has comparative advantage in all fragments relative to the standardized good when the home country knowledge reaches $K_{\max }$.

The accumulation of knowledge is assumed to be the result of learning by doing. We specify the evolution of the stock of knowledge as

$$
\dot{K}(t)=\int_{0}^{1} \varphi(s, K(t)) L_{2}(s, t) d s
$$

where $L_{2}(s, t)$ is the amount of home country labor allocated to sector $s$ at time $t$ and $\varphi(s, K) \geq$ 0 is a weighting function reflecting how output of fragment $s$ contributes to the advancement of knowledge when the current stock of knowledge is $K$. It seems natural to require that as the stock of knowledge gets larger, the outputs of simple fragments contribute less to knowledge than does output of more complex fragments. The following assumption regarding $\varphi(s, K)$ formalize this notion, and imposes the restriction that the contribution of a fragment to the accumulation of knowledge is exhausted at a finite level of knowledge. 
Assumption 2: There exists a stock of knowledge $s^{\varphi}(K)$ such that

$$
\varphi(s, K)>0 \text { for } s \geq s^{\varphi}(K) \text { and } \varphi(s, K)=0 \text { for } s<s^{\varphi}(K) \text {, where } s^{\varphi}(K) \text { is increasing }
$$

in $K$.

The learning technology described by Assumptions 1 and 2 assumes that knowledge created by production of a fragment is a public good within the sector producing good 2 . This contrasts with a case where learning is purely fragment specific and expands only as a result of output of that fragment. With the public good approach taken here, we have the possibility that output of a fragment $s$ contributes to knowledge that raises the productivity of a fragment $s^{\prime}>s$, so that $\varphi(s, K)>0$ and $b_{K}\left(s^{\prime}, K\right)<0$. This creates a form of forward knowledge linkage. Similarly, a backward knowledge linkage occurs when output of a complex fragment contributes to productivity of a simpler fragment, $\varphi\left(s^{\prime}, K\right)>0$ and $b_{K}(s, K)<0$. If $s^{b}(K)>s^{\varphi}(K)$, then fragments $s^{\prime} \in\left(s^{\varphi}(K), s^{b}(K)\right)$ will be contributing to knowledge, $\varphi\left(s^{\prime}, K\right)>0$, but not benefiting from new knowledge, $b_{K}\left(s^{\prime}, K\right)=0$. For these fragments there is a forward knowledge linkage, but no backward linkages, as illustrated by the range of $A A^{\prime}$ in Figure 2 . Similarly, if $s^{b}(K)<s^{\varphi}(K)$, there will be an interval of fragments for which there is only a backward knowledge linkage as shown by $B A^{\prime}$ in Figure 2.

Our specification can be compared with some other examples that have been used in the literature to model learning by doing with spillovers between activities. Goh and Wan (2005) use a specification that is equivalent to $b(s, K)=h(s) g(K)$ and $\varphi(s, K)=f(s)$ in our formulation. ${ }^{2}$ With this formulation, knowledge is a pure public good that raise the productivity of all fragments proportionally for all levels of knowledge. If $g(K)$ achieves its upper bound at a finite value $K_{\max }$, all fragments will exhaust the benefits of learning at the same time and $s^{b}(K)=0$ for all $K<K_{\max }$. We opt for a framework in that allows knowledge to have differential impacts across the fragments. Goh and Wan capture the increasing complexity of fragments by assuming that $f(s)$ is increasing in $s$, so that one accumulates knowledge more rapidly by producing more complex fragments. This means that the contribution of a fragment to the accumulation of knowledge will never be exhausted so that forward knowledge linkage and backward knowledge linkage always exist at the same time.

Young (1991) examines a model of learning by doing with knowledge spillovers between sectors of the economy where $b(s, K)=\bar{b} e^{-K} e^{\max [s-K, 0]}$ and $\varphi(s, K)=1$ for $s>K$ and 0 otherwise. In Young's case the stock of knowledge can be interpreted as representing the most advanced good for which learning has been exhausted. As in our analysis, the complexity of goods affects productivity growth in two ways. One is that the benefits of learning vary with $s$ : only goods that are more complex than the current state can benefit from learning by doing, and all benefit proportionally. The other is that the production of goods contributes to the stock of knowledge only if the good is more complex than the current state, and all such goods contribute equally to knowledge. These restrictions limit attention to the case in which $s^{\varphi}(K)=s^{b}(K)$.

\footnotetext{
${ }^{2}$ Specifically, Goh and Wan (2005) consider the case with $s \in\{1,2\}$ and $g(K)=e^{-K}$.
} 


\section{The Competitive Equilibrium with Trade}

We begin with the case of a competitive equilibrium with free trade, assuming that the benefits of learning are external to the firm. Under this assumption, the cost of labor to produce a unit of fragment $s$ is $w b(s, K)$ in the home country and $w^{*} b^{*}(s)$ in the foreign country, where $w$ and $w^{*}$ denote the respective wage rates. Similarly, the cost of good 1 will be $w a$ at home and $w^{*} a^{*}$ abroad.

In order to limit the number of cases to consider, we will assume that demand for good 2 is sufficiently small that both countries would produce good 1 at a free trade equilibrium. Choosing good 1 as the numeraire, we have $w=\frac{1}{a}$ and $w^{*}=\frac{1}{a^{*}}$. The home country will produce all fragments for which $w b(s, K) \leq w^{*} b^{*}(s)$. Assumption 1a then implies that the home produces all goods for which $s \in[0, m]$, where the condition for the marginal fragment is

$$
w b(m, K)=w^{*} b^{*}(m) .
$$

We can invert $(2)$ to obtain $\tilde{m}(K)$, where differentiating $(2)$ yields

$$
\frac{d \tilde{m}}{d K}=-\left(\frac{\frac{b_{K}(m, K)}{b(m, K)}}{\frac{b_{s}(m, K)}{b(m, K)}-\frac{b_{s}^{*}(m)}{b^{*}(m)}}\right) .
$$

The denominator of (3) is positive under assumption 1a, and the numerator is negative as long as $\tilde{m}(K)>s^{b}(K)$. If the marginal fragment has not achieved its maximum productivity level, then increases in the stock of knowledge will raise the productivity of the home country for its marginal fragment and expand the range of fragments produced in the home country. This is illustrated in Figure 2, which shows the $\tilde{m}(K)$ locus. The $\tilde{m}$ locus will be increasing in $K$ for $\tilde{m}(K)>s^{b}(K)$ and will be independent of $K$ for $\tilde{m}(K)<s^{b}(K)$. Assumption 1c guarantees that $m\left(K_{0}\right) \in(0,1)$. Figure 2 illustrates a case where $\frac{b(1, \bar{K}(1))}{b^{*}(1)}>\frac{a}{a^{*}}$, so the foreign country would continue to have comparative advantage in the most complex fragment even if the home country attains its maximum stock of knowledge.

The pattern of specialization given by (2) can be combined with (1) to characterize dynamics of the trade pattern in a free trade equilibrium. The price of a unit of good 2 will be the sum of the costs of the individual fragments,

$$
p(K)=\int_{0}^{\tilde{m}(K)} w b(s, K) d s+\int_{\tilde{m}(K)}^{1} w^{*} b^{*}(s) d s
$$

Worldwide consumption of good 2 will be $C_{2}^{W}=D(p)$, where $D(p)=\left(u^{\prime}\right)^{-1}(p)+\left(u^{*^{\prime}}\right)^{-1}(p)$. Letting $s^{\varphi}(K) \equiv \bar{K}^{\varphi^{-1}}(K)$, only the fragments in the interval $\left[s^{\varphi}(K), \tilde{m}(K)\right]$ will contribute to the accumulation of knowledge. The demand for labor to produce a unit of fragment $s$ is $D(p(K)) b(s, K)$, so we can substitute into (1) to solve for the evolution of the stock of knowledge at time $t$ in a competitive equilibrium 


$$
\dot{K}(t)=D(p(t)) \int_{s^{\varphi}(K(t))}^{\tilde{m}(K(t))} \varphi(s, K(t)) b(s, K(t)) d s
$$

The stock of knowledge will continue to grow as long as $s^{\varphi}(K(t))<\tilde{m}(K(t))$.

Additions to the stock of knowledge in the home country reduce the cost of producing fragments at home. From (3), the range of fragments produced by the home country will continue to expand if $-b_{K}(m, K) \dot{K}>0$, which requires both that production is generating positive learning benefits (i.e. $\dot{K}>0$ ) and that the learning benefits the marginal fragment (i.e. $\left.b_{K}(m, K)<0\right)$. In order for production to generate learning benefits, the marginal good must be contributing to knowledge creation, which requires that $s^{\varphi}(K)<m$ from (5), so that the most complex fragments being produced at home are providing learning benefits. The condition of $b_{K}(m, K)<0$ requires that $s^{b}(K)<m$, that is, the marginal fragment benefit from the accumulation of knowledge. These observations can be used to establish the following result:

Proposition 1: In a free trade equilibrium where learning is external to the firm, knowledge will accumulate until it reaches $K^{C}$, where $s^{\varphi}\left(K^{C}\right)=\tilde{m}\left(K^{C}\right)$. The range of fragments produced at home will continue to expand until the level of knowledge at which $\tilde{m}(K)=$ $\min \left[s^{\varphi}\left(K^{C}\right), s^{b}\left(K^{C}\right), 1\right]$.

a) If $s^{\varphi}\left(K^{C}\right) \leq s^{b}\left(K^{C}\right)$, then $b_{K}\left(\tilde{m}\left(K^{C}\right), K^{C}\right)=0$ and the marginal good achieves its maximum possible productivity in the steady state.

b) If $s^{\varphi}\left(K^{C}\right)>s^{b}\left(K^{C}\right)$, then $b_{K}\left(\tilde{m}\left(K^{C}\right), K^{C}\right)<0$ and the marginal good does not achieve its maximum possible productivity in the steady state.

Proposition 1 can be illustrated using Figure 2. The home country stock of knowledge will continue to expand as long as $\tilde{m}(K)>s^{\varphi}(K)$, which means that the $m$ and $K$ will move along the $\tilde{m}(K)$ locus to the point where it intersects the $s^{\varphi}(K)$ locus. The dotted $s_{0}^{\varphi}(K)$ locus illustrates a case where $s_{0}^{\varphi}(K)>s^{b}(K)$, which yields a home country steady state stock of knowledge at $K_{0}^{C}$. In this case the steady state stock of knowledge does not reach the value at which the productivity of the marginal is maximized. Reaching the stock of knowledge at which the home country productivity in $\tilde{m}\left(K_{1}^{C}\right)$ is maximized would require the production of more complex fragments in which the home country is not competitive, because in this case there are only backward knowledge linkages in the steady state.

The dashed $s_{1}^{\varphi}(K)$ locus illustrates a case where $s_{1}^{\varphi}(K)<s^{b}(K)$, which results in a steady state knowledge stock of $K_{1}^{C}$. Note however that the range of fragments produced at home does not continue to expand beyond the point at which $\tilde{m}(K)=s^{b}(K)$, because the home country does not have comparative advantage in more complex fragments even when their maximum productivity is achieved. In this case, there are only forward knowledge linkages in the steady state.

In the competitive equilibrium, the range of fragments produced by the home country will be non-decreasing over time. The price of good 2 will also be non-increasing from (4). Our assumption that good 1 is produced in both countries means that wage rates will be 
unaffected by the accumulated knowledge, so the gains from technical progress will be passed on to consumers in the free trade equilibrium.

\section{The Social Planner's Problem}

We can contrast the allocation of labor in the competitive equilibrium with that which would be chosen by a social planner whose objective is to maximize world welfare. This solution will represent the outcome in a competitive equilibrium if the gains from learning are internal to the firm. If returns are not captured by the firm, then this outcome can be used to identify the subsidies required to attain the world welfare maximizing pattern of production and consumption.

World welfare can be expressed as

$$
U^{W}=\int_{0}^{\infty}\left[C_{1}^{W}+u^{W}\left(C_{2}^{W}\right)\right] e^{-\rho t} d t
$$

where $u^{W}\left(C_{2}^{W}\right)=\max _{C_{2}^{*}} u\left(C_{2}^{W}-C_{2}^{*}\right)+u^{*}\left(C_{2}^{*}\right)$. The planner will choose the time paths for employment to maximize (6) subject to the full employment constraints,

$$
\begin{aligned}
L & =L_{1}(t)+\int_{0}^{1} L_{2}(s, t) d s \\
L^{*} & =L_{1}^{*}(t)+\int_{0}^{1} L_{2}^{*}(s, t) d s
\end{aligned}
$$

the technological constraints

$$
\begin{aligned}
C_{1}(t) & \leq \frac{L_{1}(t)}{a}+\frac{L_{1}^{*}(t)}{a^{*}} \\
C_{2}(t) & \leq \frac{L_{2}(s, t)}{b(s, K(t))}+\frac{L_{2}^{*}(s, t)}{b^{*}(s)}
\end{aligned}
$$

and the knowledge accumulation condition (1).

Letting $\mu$ denote the costate variable associated with the stock of knowledge, the optimal production pattern is for the home country to produce fragments for which

$$
(w-\mu(t) \varphi(s, K(t))) b(s, K(t)) \leq w^{*} b^{*}(s)
$$

The cost of labor in the home country can be interpreted as being equal to the wage rate less the value of the knowledge created by employment in a fragment. Letting $\theta \equiv \frac{\mu \varphi(s, K)}{(w-\mu(t) \varphi(s, K))}$ be the ratio of the knowledge benefits to the net cost of labor, the social optimum will call for the home country to produce all fragments $s \in[0, M]$ if

$$
\Delta(s, K) \equiv\left(\frac{b_{s}(s, K)}{b(s, K)}-\frac{b_{s}^{*}(s)}{b^{*}(s)}-\theta \frac{\varphi_{s}(s, K)}{\varphi(s, K)}\right)>0
$$


is satisfied for any $M$ at which (9) holds with equality. The sum of the first two terms in parentheses is positive by assumption 1a, and corresponds to the requirement for a unique marginal fragment in the competitive case. The last term is the effect of an increase in complexity on learning by doing. The condition for a unique solution is that the effect of learning not increase too rapidly in $s$.

Assuming that (10) is satisfied, we can solve for a unique marginal fragment $\tilde{M}(\mu, K)$ at which (9) holds with equality. We can obtain the effect of $\mu$ and $K$ on the marginal good by differentiating this condition,

$$
\frac{\partial \tilde{M}}{\partial \mu}=\frac{\theta}{\mu \Delta(M, K)}>0 \quad \frac{\partial \tilde{M}}{\partial K}=-\frac{\left(\frac{b_{K}(M, K)}{b(M, K)}-\theta \frac{\varphi_{K}(M, K)}{\varphi(M, K)}\right)}{\Delta(M, K)}
$$

An increase in the value of knowledge will increase the range of fragments produced in the home country as long as the marginal good is contributing to the stock of knowledge, $\varphi(M, K)>0$. An increase in the stock of knowledge has two effects on the range of goods at home. One is the effect of an increase in the stock of knowledge on the productivity of the marginal good, which will tend to expand the range of fragments produced for $M>s^{b}(K)$. The second is the effect of an increase in the stock of knowledge on the learning benefits obtained from the marginal fragment. If $\varphi_{K}(M, K)<0$, more knowledge reduces the learning benefits obtained from the marginal good, tending to reduce the range of goods produced at home. In this case the two effects are opposing and the effect of an increase in knowledge on $M$ is ambiguous. If $\varphi_{K}(M, K)>0$, the two effects work in the same direction and $\frac{\partial \tilde{M}}{\partial K}>0$.

The socially optimal level of consumption will satisfy $C_{2}^{W}=D(P)$, where

$$
P=\int_{0}^{\tilde{M}(\mu, K)}(w-\mu \varphi(s, K)) b(s, K) d s+\int_{\tilde{M}(\mu, K)}^{1} w^{*} b^{*}(s) d s
$$

If the learning effects are external to the firm, then implementation of the socially optimal production pattern can be achieved with a production subsidy of $\sigma(s, t)=\mu(t) \varphi(s, K(t)) b(s, K(t))$. For a given $K(t)$, the socially optimal allocation will imply a greater range of fragments produced in the home country and a greater world consumption of good 2 than would arise in the competitive equilibrium if $\mu(t)>0$.

Figure 3 shows the relationship between the state variables and the marginal fragment produced at home. The $\tilde{M}(0, K)$ locus coincides with the $\tilde{m}(K)$ locus from the competitive equilibrium, since in this case the accumulation of knowledge has no value. For $\mu>0$, $\tilde{M}(\mu, K)>\tilde{M}(0, K)$ for $M>s^{\varphi}(K)$ and $\tilde{M}(\mu, K)=\tilde{M}(0, K)$ for $M<s^{\varphi}(K)$. This illustrates that the range of fragments produced at home will exceed those in the competitive equilibrium as long as learning has value and the marginal fragment is contributing to learning.

The evolution of the state and costate variables in the socially optimal production plan 
will be given by

$$
\begin{aligned}
\dot{K}(t) & =D\left(P(\mu(t), K(t)) \int_{s^{\varphi}(K(t))}^{\tilde{M}(\mu(t), K(t))} \varphi(s, K(t)) b(s, K(t)) d s\right. \\
\dot{\mu}(t) & =\left(\rho-\frac{\partial \dot{K}}{\partial K}\right) \mu(t)+\int_{s^{b}(K(t))}^{\tilde{M}(\mu(t), K(t))}(w-\mu(t) \varphi(s, K(t))) \frac{b_{K}(s, K)}{b(s, K)} L_{2}(s) d s
\end{aligned}
$$

The accumulation of knowledge is obtained by evaluating (1) at the optimal production plan. The change in the value of the costate variable can be interpreted as the difference between the cost of rental cost of knowledge capital and the marginal product of knowledge capital on the optimal path. The last term of (14) is the change in cost resulting from an increment of knowledge at time $t$, which is the marginal product of knowledge capital. The second term in parentheses is the impact of an increment of knowledge on the productivity of learning, which has an effect similar to the rate of depreciation.

\subsection{Dynamics of the Socially Optimal Plan}

We begin by characterizing the steady state for the social planner's problem, and showing that the steady state of the social planner's problem coincides with that from the competitive equilibrium in Proposition 1.

A steady state for the social planner's problem will exist if there is a pair $\left\{\mu^{S}, K^{S}\right\}$ such that (13) and (14) are satisfied with $\dot{\mu}=\dot{K}=0$. From (13), $\dot{K}=0$ if $\tilde{M}(\mu, K)=s^{\varphi}(K)$. Note however that since $\varphi\left(s^{\varphi}(K), K\right)=0$, we have from (9) that this condition is equivalent to $\tilde{m}(K)=s^{\varphi}(K)$. Therefore, the steady state of the social planner's problem will coincide with that of the competitive equilibrium. To determine the steady state value of $\mu$, consider first the case where $\tilde{m}\left(K^{S}\right)=s^{\varphi}\left(K^{S}\right)<s^{b}\left(K^{S}\right)$, so that there are only forward linkages at the steady state. The marginal fragment would not benefit from additional learning by doing at the socially optimal steady state, so the second term in (14) will be 0 . In this case, $\dot{\mu}=0$

requires $\mu=0$. If $\tilde{m}\left(K^{S}\right)=s^{\varphi}\left(K^{S}\right)>s^{b}\left(K^{S}\right)$, on the other hand, the fragments in the interval $\left[s^{b}\left(K^{S}\right), \tilde{m}\left(K^{S}\right)\right]$ have not achieved their highest productivity.

To be added.

\section{References}

[1] Antras, Pol and Elhanan Helpman, "Global Sourcing," Journal of Political Economy 112 (2004):552-580.

[2] Bond, Eric W., Ronald W. Jones and Ping Wang, "Economic Takeoffs in a Dynamic Process of Globalization," Review of International Economics 13 (2005):1-19.

[3] - "Market linkages with fragmented production," North American Journal of Economics and Finance 16 (2005): 119-35. 
[4] - "Input Quality, Relational Contracts And International Outsourcing," $P a-$ cific Economic Review 13 (2008): 391-404.

[5] Dei, Fumio, "Peripheral Tasks Are Offshored," Review of International Economics 18 (2010):807-17.

[6] Feenstra, Robert, "Integration of Trade and the Disintegration of Production in the Global Economy," Journal of Economic Perspectives 12 (1998):31-50.

[7] Goh, Ai-Ting and Henry Y. Wan, Jr (2005), "Fragmentation, Engel's Law, and Learning," Review of International Economics 13 (2005):518-28.

[8] Golub, Stephen, Ronald Jones, and Henryk Kierzkowski, "Globalization and CountrySpecific Service Links", The Journal of Economic Policy Reform 10 (2007):63-88.

[9] Grossman, Gene M. and Esteban Rossi-Hansberg, "Trading Tasks: A Simple Theory of Offshoring," American Economic Review 98 (2008): 1978-97.

[10] $\longrightarrow$ and "Task Trade between Similar Countries," Available at http://www.princeton.edu/ ${ }^{\sim}$ grossman/grossman_working_papers.htm (2011) unpublished.

[11] Jones, Ronald and Henry Kierzkowski, "The Role of Services in Production and International Trade: A Theoretical Framework," ch. 3 in Jones and Anne Krueger (eds.), The Political Economy of International Trade, Blackwells (1990).

[12] and "A Framework For Fragmentation", Chapter 2 in Arndt and Kierzkowski (eds.), Fragmentation: New Production Patterns in the World Economy, Oxford University Press (2001).

[13] Ngienthi, Wanida and Fumio Dei, "The Impact of Differential Falls in Offshoring Costs on Welfare," Review of International Economics (2011): forthcoming.

[14] Ngienthi, Wanida, Yan Ma, and Fumio Dei, "Supermodularity and Global Supply Chains without the South," Review of International Economics (2011): forthcoming. 


\section{Appendix}

To analyze this problem, we form the current value Hamiltonian

$$
\begin{aligned}
H= & V\left(C_{1}^{W}, C_{2}^{W}\right)+\mu \int_{0}^{1} \varphi(s, K) L_{2}(s) d s+\lambda\left(L-L_{1}-\int_{0}^{1} L_{2}(s) d s\right)+\psi_{1}\left(\frac{L_{1}}{a}+\frac{L_{1}^{*}}{a^{*}}-C_{1}^{W}\right) \\
& +\lambda^{*}\left(L^{*}-L_{1}^{*}-\int_{0}^{1} L_{2}^{*}(s) d s\right)+\left(\int_{0}^{1} \psi_{2}(s)\left(\frac{L_{2}(s)}{b(s, K)}+\frac{L_{2}^{*}(s)}{b^{*}(s)}-C_{2}^{W}\right)\right) d s
\end{aligned}
$$

The necessary conditions for choice of labor in sector 1 and consumption of good 1 are $\frac{\partial H}{\partial L_{1}}=-\lambda+\frac{\psi_{1}}{a} \leq 0, \frac{\partial H}{\partial L_{1}^{*}}=-\lambda^{*}+\frac{\psi_{1}}{a^{*}} \leq 0$, and $\frac{\partial H}{\partial C_{1}^{W}}=1-\psi_{1} \leq 0$, with equality if the respective values are positive. The assumption that labor is sufficiently abundant in each country implies $\psi_{1}=1, \lambda=\frac{1}{a}=w$, and $\lambda^{*}=\frac{1}{a^{*}}=w^{*}$. Using these results in the necessary conditions for choice of labor for fragment $s$ yields

$$
\begin{aligned}
-w^{*}+\frac{\psi_{2}(s)}{b^{*}(s)} & \leq 0 \\
-w+\frac{\psi_{2}(s)}{b(s, K)}+\mu \varphi(s, K) & \leq 0
\end{aligned}
$$

Since at least one of these must hold with equality in order for good 2 to be produced, we have $\psi_{2}(s)=\min \left[w^{*} b^{*}(s),(w-\mu \varphi(s, K)) b(s, K)\right]$, which yields (9) in the text. The necessary condition for choice of $C_{2}^{W}$ yields $u^{W^{\prime}}\left(C_{2}^{W}\right)=\int_{0}^{1} \psi_{2}(s) d s$, which yields the consumer price in the socially optimal case given by (12).

The evolution of the costate variable is determined by $\dot{\mu}=\rho \mu-\frac{\partial H}{\partial K}$, which yields (14).

The transversality condition requires $\lim _{t \rightarrow \infty} \mu(t) e^{-\rho t}=0$. 